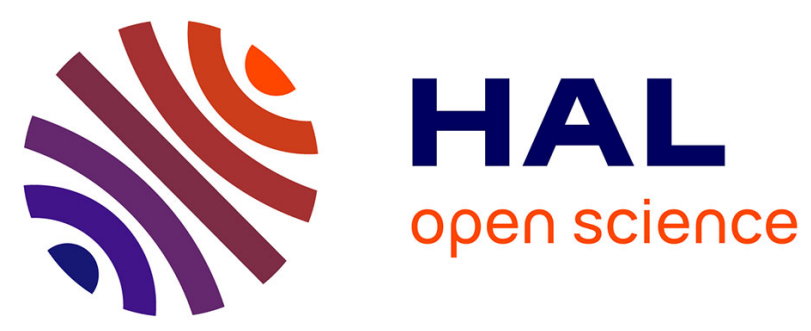

\title{
Paraxial Gaussian beam shooting algorithm for 3D propagation simulation in built environments
}

Emna-Amira Fnaiech, Christine Letrou, Arnaud Ginestet, Gilles Beauquet

\section{To cite this version:}

Emna-Amira Fnaiech, Christine Letrou, Arnaud Ginestet, Gilles Beauquet. Paraxial Gaussian beam shooting algorithm for 3D propagation simulation in built environments. ICEAA 2015: International Conference on Electromagnetics in Advanced Applications, Sep 2015, Torino, Italy. pp.1155 - 1158, 10.1109/ICEAA.2015.7297300 . hal-01359513

\section{HAL Id: hal-01359513 https://hal.science/hal-01359513}

Submitted on 2 Sep 2016

HAL is a multi-disciplinary open access archive for the deposit and dissemination of scientific research documents, whether they are published or not. The documents may come from teaching and research institutions in France or abroad, or from public or private research centers.
L'archive ouverte pluridisciplinaire HAL, est destinée au dépôt et à la diffusion de documents scientifiques de niveau recherche, publiés ou non, émanant des établissements d'enseignement et de recherche français ou étrangers, des laboratoires publics ou privés. 


\section{Paraxial Gaussian beam shooting algorithm for 3D propagation simulation in built environments}

\author{
E.A. Fnaiech* \\ C. Letrou $^{\dagger}$
}

\begin{abstract}
Frame-based Gaussian Beam Shooting is presented as an alternative to ray-based methods, when reliable and accessible knowledge of propagated electromagnetic fields is required at all points in built-up environments.

This communication presents a general Gaussian Beam Shooting algorithm based on paraxial beam tracking complemented with the use of "image" frames and with frame redecomposition on reflecting surfaces, to account for the presence of ground and buildings.

Numerical results obtained in the case of a "single wall" and of a "parallelipedic building" are compared to Parabolic Equation and FEKO software results.
\end{abstract}

\section{INTRODUCTION}

Reliable and accessible knowledge of propagated electromagnetic fields at all points in built-up environments is a prerequisite to control emission levels and optimize spectrum management in communication systems, as well as to optimize the design of ground-based Radar systems taking advantage of multipath propagation.

Gaussian Beam Shooting (GBS) is well suited for 3D field simulations in multi-reflecting contexts, thanks to spectral localization of Gaussian beams (GB), allowing for paraxial approximations of reflected fields, and subsequent tracking algorithms, similar to ray-based ones. In the millimetric range, a "paraxial tracking" GBS algorithm was validated by comparison with experimental results, in indoor environments [1]. As compared to ray launching or ray tracing methods, GBS does not rely on any far field assumption, and in the millimetric range paraxial tracking appears to be accurate enough regarding diffraction, thus limiting the number of launched beams with respect to launched or traced rays in ray algorithms.

At frequencies of conventional communication or ground based Radar systems, however, paraxial GB tracking does not account for diffraction ef-

*Télécom SudParis (Lab. SAMOVAR - UMR CNRS 5157), 9 rue Charles Fourier, 91011 Evry Cedex, France

†Télécom SudParis (Lab. SAMOVAR - UMR CNRS 5157), 9 rue Charles Fourier, 91011 Evry Cedex, France, e-mail: christine.letrou@telecom-sudparis.eu, tel.: +33 1607646 29, fax: +33160764433

${ }^{\ddagger}$ NOVELTIS, 153 rue du Lac, 31670 Labege, France

$\S$ THALES Air Systems S.A., Hameau de Roussigny, 91470 Limours, France

\author{
A. Ginestet ${ }^{\ddagger}$ \\ G. Beauquet ${ }^{\S}$
}

fects with reasonable accuracy. Some recent studies have proposed "beam to beam" formulations to discretize radiated and scattered fields into sets of GBs: empirical sampling and normalisation at arrival [2], empirical sampling in the angular domain and frame decomposition along the edge [3], frame decomposition and frame based truncation of fields on obstacle surface [4]. None of these formulations is used in this study: frame decomposition is favored, and direct frame decomposition of incident fields on reflecting or equivalence surfaces is performed when needed.

Section 2 is devoted to an outline of the frame based GBS algorithm used in this work, which combines free space GBS with:

- frame decompositions of source and incident field distributions,

- the use of image frames to perform paraxial GB tracking in the presence of reflecting surfaces,

- "beam selection" to determine which beams are incident on a given obstacle and on its edges.

Section 3 presents results obtained by GBS over a reflecting ground, in the presence of a screen and of a single building. Comparisons with Parabolic Equation simulations [5], and Method of Moments or PTD results are shown.

\section{FRAME BASED GAUSSIAN BEAM SHOOTING ALGORITHM}

In order to represent fields radiated into a halfspace as superpositions of Gaussian beams (GB), complete sets of Gaussian windows defined in the source plane are used to discretize source field distributions. Such sets belong to the class of "frames" in $L_{2}\left(\mathbb{R}^{2}\right)$.

\subsection{Frame of Gaussian windows}

The frame windows $\Psi_{\boldsymbol{\mu}}(y, z)$ used to describe a planar source distribution in the $y O z$ plane are constructed as the product of frame windows $\psi_{m, n}^{y}(y)$ and $\psi_{p, q}^{z}(z)$ in $L_{2}(\mathbb{R})$ :

$$
\Psi_{\boldsymbol{\mu}}(y, z)=\psi_{m, n}^{y}(y) \psi_{p, q}^{z}(z)
$$

where $\boldsymbol{\mu}=(m, n, p, q)$ is a composite translation index in $\mathbb{Z}^{4}$, and $\psi_{m, n}^{u},(m, n) \in \mathbb{Z}^{2}$, is obtained by translating a Gaussian function $\psi$ along the spatial 
coordinate $u$ and along its spectral counterpart $k_{u}$ :

$$
\psi_{m, n}^{u}(u)=\psi(u-m \bar{u}) e^{i n \bar{k}_{u} u}
$$

with $\psi(u)=\left(\frac{\sqrt{2}}{L}\right)^{\frac{1}{2}} \exp \left(-\pi \frac{u^{2}}{L^{2}}\right) ; \bar{u}$ and $m$ are the spatial translation step and index, $\bar{k}_{u}$ and $n$ their spectral counterparts.

The set $\left\{\psi_{\boldsymbol{\mu}}, \mu \in \mathbb{Z}^{2}\right\}$ is a frame if and only if $\bar{u} \bar{k}_{u}=2 \pi \nu$, with $\nu<1$ ( $\nu$ : oversampling factor), and the favorite choice for the translation steps is: $\bar{u}=\sqrt{\nu} L, \bar{k}_{u}=\sqrt{\nu}(2 \pi / L)$ ("balanced" frame).

An $\alpha$-polarized electric field distribution $E^{\alpha}(y, z)$ is discretized by "frame decomposition" as:

$$
E^{\alpha}(y, z)=\sum_{\boldsymbol{\mu} \in \mathbb{Z}^{4}} A_{\boldsymbol{\mu}}^{\alpha} \Psi_{\boldsymbol{\mu}}(y, z)
$$

with the $A_{\boldsymbol{\mu}}$ complex coefficients called "frame coefficients". These coefficients are not unique, but can be calculated by projecting the function on a "dual frame" of functions [6, 7]. This calculation is performed by Discrete Fourier Transform, and for high enough oversampling $(\nu \leq 0.25)$, approximating the dual functions by Gaussian windows provides accurate enough results [7].

\subsection{Gaussian beam shooting}

Let us denote $\boldsymbol{B}_{\boldsymbol{\mu}}^{\alpha}(\boldsymbol{r})$ the field radiated at point $\boldsymbol{r}$ by an $\alpha$-polarized electric field distribution $E^{\alpha}=$ $\Psi_{\boldsymbol{\mu}}(y, z)(\alpha=y$ or $z)$. The paraxial expression of $\boldsymbol{B}_{\boldsymbol{\mu}}^{\alpha}(\boldsymbol{r})$, obtained by paraxial asymptotic evaluation of a plane wave spectrum integral, writes as:

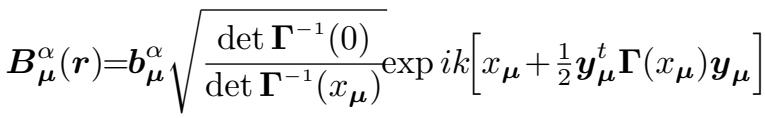

with $\boldsymbol{b}_{\boldsymbol{\mu}}^{\alpha}$ a vector depending on the source polarization. This expression is that of a GB with its axis along the $x_{\boldsymbol{\mu}}$ direction determined by the spectral translation vector $\left(n \bar{k}_{y}, q \bar{k}_{z}\right) \cdot \boldsymbol{y}_{\boldsymbol{\mu}}=\left(y_{\boldsymbol{\mu}}, z_{\boldsymbol{\mu}}\right)$ is the position vector of the point $\boldsymbol{r}$ in a plane transverse to the $x_{\boldsymbol{\mu}}$ axis, and $\boldsymbol{\Gamma}$ is a complex curvature matrix yielding transverse field decay.

The expression of the radiated field at point $\boldsymbol{r}$ in the $x>0$ half-space is then easily derived using (2), in the form of a GBS formula:

$$
\boldsymbol{E}(\boldsymbol{r})=\sum_{\boldsymbol{\mu} \in \mathbb{Z}^{4}}\left[A_{\boldsymbol{\mu}}^{y} \boldsymbol{B}_{\boldsymbol{\mu}}^{y}(\boldsymbol{r})+A_{\boldsymbol{\mu}}^{z} \boldsymbol{B}_{\boldsymbol{\mu}}^{z}(\boldsymbol{r})\right]
$$

\subsection{Reflexion and diffraction by planar ob- stacles}

GBS algorithms have to take advantage of the transverse localization of beams to limit the number of beams to shoot. A selection algorithm determines which beams contribute to the field in a given zone or on a given surface [8]. In the presence of an obstacle, the same algorithm is used to determine which of the beams impinging on the obstacle surface are impinging on the edge or corners of this surface. This information leads to different processing of beams, either paraxially reflected if they do not "touch" edges, or else "diffracted".

In our algorithm, paraxial reflexion is accounted for by introducing image frame windows, with their vector part transformed by reflexion operators (Fresnel or derived from surface impedance models) [8]. Diffraction is accounted for by considering the fields incident on a given surface as being truncated at the edge of the surface. Contrary to Physical Optics however, where equivalent currents radiate into all directions of space, GBS involves plane wave spectrum representations of fields, radiating into one half-space only, and spectrally narrow GB fields can be transformed by reflexion operators applied to plane waves.

In the special case of obstacles with perfectly conducting "walls", equivalence theorems are specially easy to implement for a zero order approximation of diffraction effects. The surface of the obstacle is considered as an equivalent surface where the null tangent electric field boundary condition must be enforced, in the presence of source fields propagating in free space. This approach leads to frame decomposition of incident fields on all the virtual surfaces replacing the walls of the obstacle. The radiated field obtained by GBS from these walls is then substracted from the source field propagating in free space. Next section will illustrate the application of this algorithm.

\section{NUMERICAL RESULTS}

Test cases are designed to compare results obtained by GBS, with those of a Parabolic Wave Equation algorithm developed at NOVELTIS (3DPWE), and with those obtained with the FEKO code.

The source is given as a field distribution in the $x O z$ plane, with linear polarization along $y$ (horizontal) or $z$ (vertical). The distribution is of the form of a Gaussian frame window $\Psi_{\boldsymbol{\mu}}(y, z)$, defined by: $L=7.5 \lambda, \nu=0.16$, centered at $z=12.5 \mathrm{~m}$, $y=0$, without any spectral shift. In the following, the frequency is taken equal to $430 \mathrm{MHz}$, a classical frequency used for UHF ground Radar applications, and the source polarization is horizontal. The frame used to decompose the source distribution in the $y O z$ plane, as well as incident fields on reflecting planes, is defined by $L=10 \lambda$ and $\nu=0.16$. 


\subsection{Test case with screen}

The first test case addresses the problem of diffraction by a perfectly conducting (PEC) screen, in the presence of a plane PEC ground, as shown in figure 1 . The screen is 5 meter wide and 20 meter high, in the plane $x=200 \mathrm{~m}$, centered with respect to the vertical plane $y=0$.

In such a case, the GBS algorithm computes propagated fields by summing up six different fields, each of them obtained by GB summation:

- the source beam field and its image field with respect to the ground, both radiated in free space (without screen) into the half-space $x>0$.

- the field reflected by the screen, obtained by GBS from the frame decomposition of the field incident on the screen, which is radiated by the source and by its image by the ground.

- the image of this reflected field by the ground; both reflected fields are present in the half-space $x<200 \mathrm{~m}$.

- the "shadow" field, radiated into the half-space $x>200 \mathrm{~m}$ by the opposite of the field incident on the screen; the image of this "shadow" field by the ground.

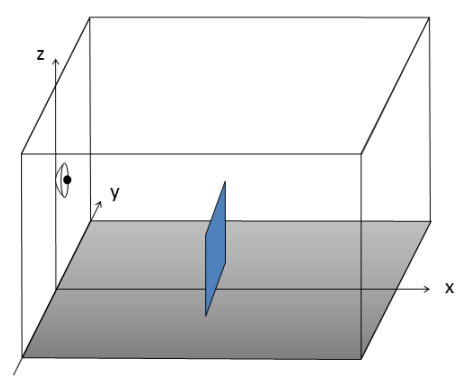

Figure 1: Test case with a vertical screen at $x=200 \mathrm{~m}$, on a flat ground.

A comparison with MoM results obtained with FEKO and with results obtained with 3DPWE is presented in Figures 2 and 3 along vertical lines at distances $x=100 \mathrm{~m}$ and $300 \mathrm{~m}$ in the $x O z$ plane (100 $\mathrm{m}$ in front of and $100 \mathrm{~m}$ behind the screen). Results visibly compare well, in spite of strong oscillations along the $x$ axis in the region where reflected and incident fields superpose (cf Figure 6).

\subsection{Test case with building}

This case is derived from the previous one, the screen being replaced by a "building" modelled by a PEC parallelepipoid, with $5 \times 5 \mathrm{~m}^{2}$ basis and 20 meter height, with its first wall parallel to the $y O z$ plane, at $x=200 \mathrm{~m}$ (Figure 4 ).

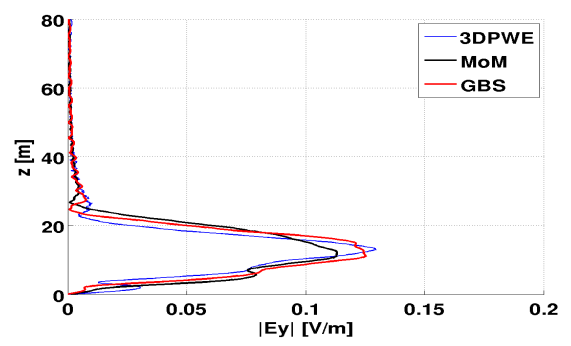

Figure 2: Horizontal field component along the line $y=0, x=100 \mathrm{~m}$.

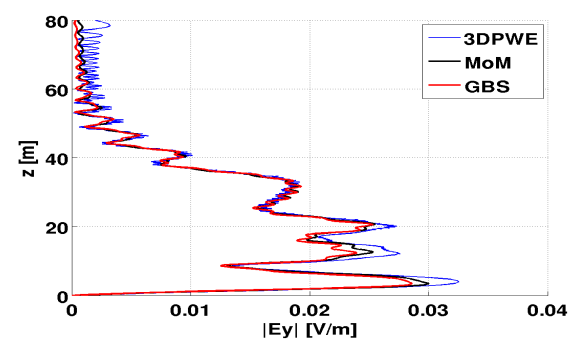

Figure 3: Horizontal field component along the vertical lines in $x O z$ plane, at $x=300 \mathrm{~m}$.

Propagated fields are shown in Figure 5 on a horizontal map parallel to $x O y$ at height $z=12.5 \mathrm{~m}$. Fields are sampled every 1 meter along $x, 0.25$ meter along $y$. The building is colored in black at the center of the map. Oscillations caused by intereferences between incident and reflected waves $(x<200 \mathrm{~m})$, as well as shadowing for $x>200 \mathrm{~m}$ and diffraction effects are clearly visible.

A comparison with PTD results obtained with FEKO and with results obtained with $3 \mathrm{DPWE}$ is presented in Figure 6 along the horizontal line defined by $y=0$ and $z=12.5 \mathrm{~m}$. Strong oscillations in front of the building do not allow for a clear comparison of the different results, which vary however in the same range of values. Behind the building, GBS and PTD results match very well.

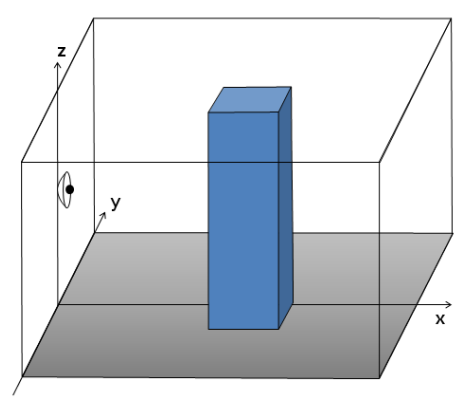

Figure 4: Test case with a parallelepipedic building at $x=200 \mathrm{~m}$, on a flat ground. 


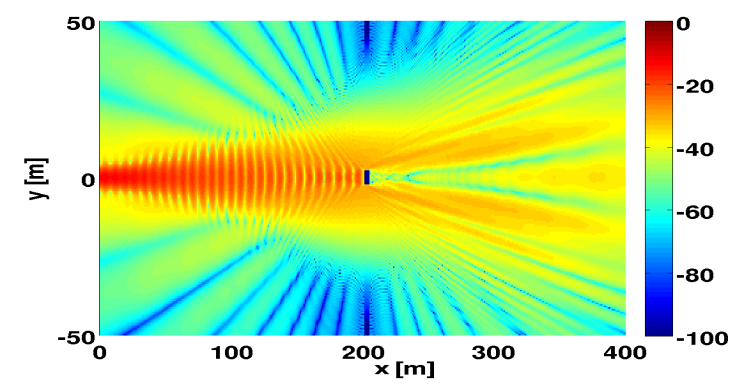

Figure 5: Horizontal field component in the horizontal plane $z=12.5 \mathrm{~m}$, in the presence of the building.

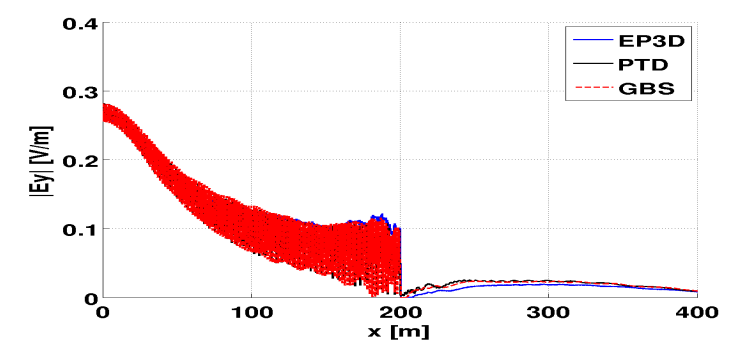

Figure 6: Horizontal field component along the beam axis: comparison between 3DPWE, PTD and GBS.

\section{CONCLUSION}

Computation of propagated fields by a frame-based Gaussian beam shooting algorithm has been performed in test case environments involving reflecting/diffracting obstacles. Diffraction is accounted for in an approximate way, through frame redecomposition on reflecting surfaces. Numerical results compare very well with results obtained with the FEKO code, through a Method of Moments in the case involving a single screen, through PTD in the case of a single building. Results involving both types of obtacles, as a model for a realistic non line of sight situation, will be presented at the conference.

\section{Acknowledgment}

The authors acknowledge partial support by the French National Research Agency (ANR) under "Specific Support for Research Works and Innovation Defense" (ASTRID) grant ANR-12-ASTR0013.

\section{References}

[1] A. Fluerasu and C. Letrou, "Gaussian beam launching for 3D physical modeling of propagation channels," Annales des télécommunications- Annals of telecommunications, vol. 64, no. 11-12, pp. $763-776$, december 2009.

[2] J. M. Darras, T. George, and P. Pouliguen, "Diffraction by a sharp edge for an electromagnetic field described by summation of gaussian beams: the Ufimtsev way," in Progress in Electromagnetics Research Symposium (PIERS 2012), Moscow, Russia, August 2012.

[3] M. Katsav and E. Heyman, "Gaussian beam summation representation of beam diffraction by an impedance wedge: a 3D electromagnetic formulation within the Physical Optics approximation," IEEE Trans. Antennas Propagat., vol. 60, no. 12, pp. 5843-5858, Dec. 2012.

[4] I. Ghannoum, C. Letrou, and G. Beauquet, "Frame based Gaussian beam bouncing," in EMTS'10 : 20th International Symposium on Electromagnetic Theory, IEEE, Ed., 2010, pp. $68-71$.

[5] M. Levy, Parabolic equation methods for electromagnetic wave propagation. Electromagnetic waves series 45. The institution of Electrical Engineers, 2000.

[6] D. Lugara and C. Letrou, "Printed antennas analysis by a Gabor frame-based method of moments," IEEE Trans. Antennas Propagat., vol. 50, no. 11, pp. 1588-1597, 2002.

[7] A. Shlivinski, E. Heyman, A. Boag, and C. Letrou, "A phase-space beam summation formulation for ultrawide-band radiation," IEEE Trans. Antennas Propagat., vol. 52, no. 8, pp. 2042-2056, 2004.

[8] E. Fnaiech, C. Letrou, A. Ginestet, and G. Beauquet, "Frame-based gaussian beam shooting simulation of back-scattered fields in the presence of a building," in Electromagnetics in Advanced Applications (ICEAA), 2014 International Conference on, Aug 2014, pp. 484-487. 